\title{
Immunophenotyping of primary and metastatic lesions in feline mammary tumors - are they equal?
}

\author{
M. Soares*, J. Correia**, A. Murta*** and F. Ferreira* \\ *CIISA, Departamento de Morfologia e Função, Faculdade de Medicina Veterinária, Universidade Técnica de \\ Lisboa, Portugal, Av. Universidade Técnica, 1300-477 Lisboa, Portugal. \\ **CIISA, Departamento de Sanidade Animal, Faculdade de Medicina Veterinária, Universidade Técnica de \\ Lisboa, Portugal, Av. Universidade Técnica, 1300-477 Lisboa, Portugal. \\ ***Hospital Escolar, Faculdade de Medicina Veterinária, Universidade Técnica de Lisboa, Portugal, Av. \\ Universidade Técnica, 1300-477 Lisboa, Portugal.
}

The identification of genome-wide expression profiles that discriminate different molecular subtypes of diseases became a recent field of research, with a visible impact in cancer research. In breast cancer, molecular immunophenotyping of the tumor is critical to support therapeutic decisions [1].

Over the last years, some studies revealed that a high percentage of Feline Mammary Tumours (FMTs) display fHER2 overexpression followed by a very short survival period, like in woman HER2-positive breast cancer [2,3]. Usually, FMTs are malignant and very aggressive (85\% to 93\%), showing bad prognosis. In feline oncology, the histopathological classification is the only possible option, meaning that little data about molecular expression profiles is available. Given these facts, our study aims to confirm if primary tumor immunophenotype is conserved at regional and distant metastasis.

Two primary mammary carcinomas, their regional and distant metastasis were collected during necropsy examination of two queens. The immunohistochemical analysis measured HER2 status, progesterone receptor (PR), estrogen receptor (ER), cytokeratin (CK) expression levels and the Ki67 index in all samples using the antibodies: anti-HER2 (A0485, DAKO); anti-PR (1E2, Ventana); anti-ER (SP1, Ventana); anti-CK (AE1/AE3, DAKO) and anti-Ki67 (MM1, Leica). The fixation and the interpretation criteria followed the American Society of Clinical Oncology guidelines.

The first tumor was an anaplastic mammary carcinoma with high malignancy grade (III) both in the primary tumor and in metastatic lesions (Fig.1). Immunophenotyping revealed HER2 and PR positivity in both primary mass and regional metastasis (Fig.1 - A4, B4; A5, B5), but ER negative staining in these locations (Fig.1- A6, B6). Distant metastasis showed a lack of PR expression (Fig.1 - C5), but maintained equal status of the two other markers (Fig.1 - C4, C6). Similar Ki-67 index values were determined in primary tumor (0.26) and in metastasis (0.19). The second FMT was a tubulopapillary carcinoma (Fig.2) with high malignancy grade (III) and, unlike, the first tumor, immunolabeling for PR was positive both in the primary tumor and in the different metastasis, revealing molecular pattern conservation. The Ki-67 index was always high either in primary tumor $(0.33)$ as in regional (0.48) and distant metastases (0.41) (Fig.2 - A3, B3, C3). All tissue samples were positive for CK, confirming their epithelial nature (Fig.1 - A2, B2, C2 and Fig.2 - A2, B2, C2).

Our study points out that FMTs molecular expression patterns may not be preserved in primary tumor and metastatic lesions. If we just looked to the immunophenotype of the primary mass and the regional metastasis, both carcinomas would be classified as luminal B subtype (ER-, PR+ and HER2+). However, when we evaluated the distant metastasis, while the immunophenotype of the second FMT was conserved, the PR expression in the first FMT was lost, which implied a tumor reclassification to HER2 subtype. In women, both subtypes have anthracycline-based regimens prescription but luminal B subtype also benefits with hormonal therapy, while the HER-2 subtype has indication for specific immunotherapy [4]. Similarly to women breast cancer, the molecular classification of feline mammary tumors should be performed to improve treatmentresponse and animal live quality. 
References:

1. Kim S., et al., Biology Direct, 7:21, 2012.

2. DeMaria R. et al., Cancer Res., 65:907, 2005.

3. Millanta F., et al., Vet. Pathol., 42:30, 2005.

4. Goldhirsch A., et al., Ann. Oncol. 22: 1736, 2011.
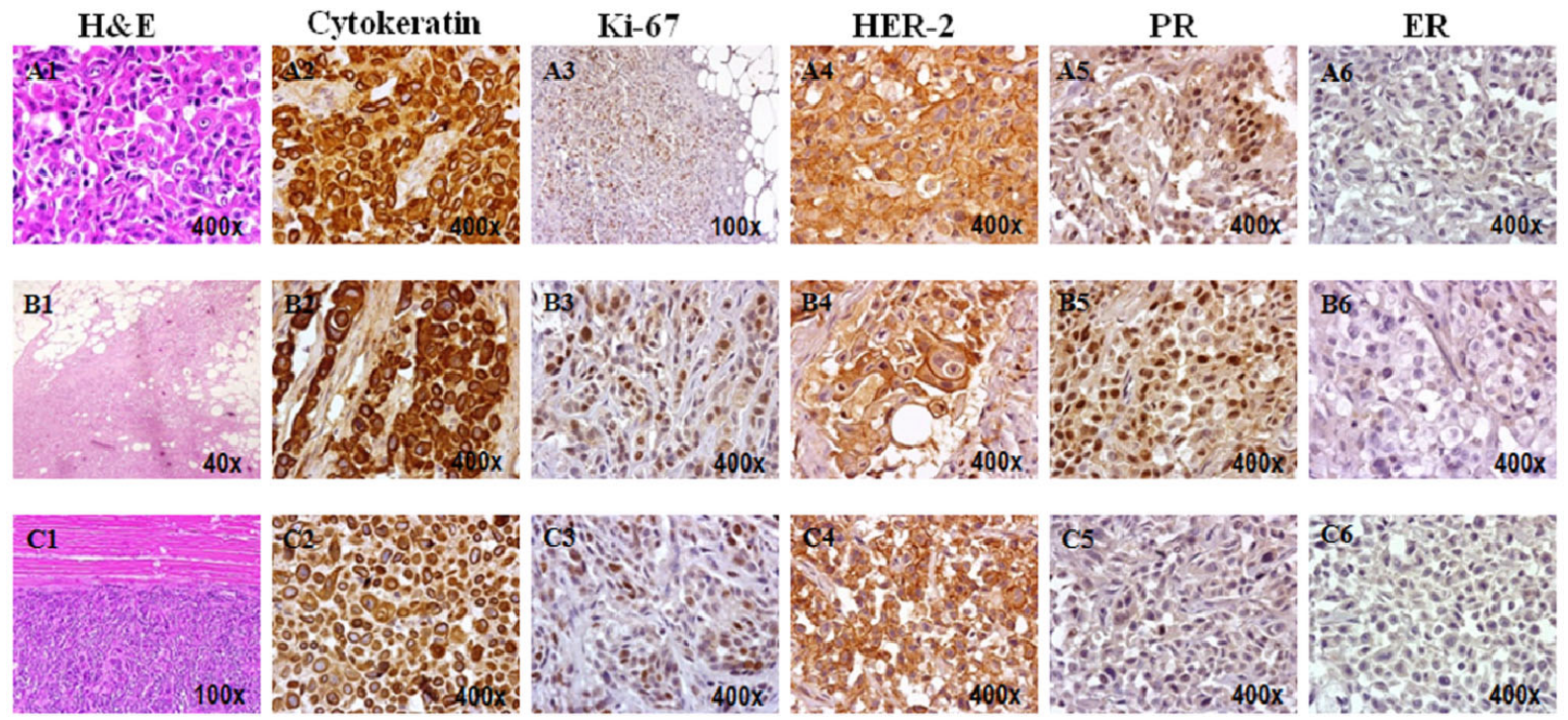

Figure 1. Immunophenotype of a feline mammary anaplastic carcinoma: primary tumor (A1-A6), regional metastasis (B1-B6) and distant metastasis (C1-C6). A2, B2, C2 - Strong membrane CK immunolabeling; A3, B3, C3 - Ki-67 nuclear immunostaining; A4, B4, C4 - HER-2 positive status; A5 and B5 - PR positive immunostaining; C5 - PR negative immunostaining in distant metastases; A6, B6, C6 - ER negative staining.
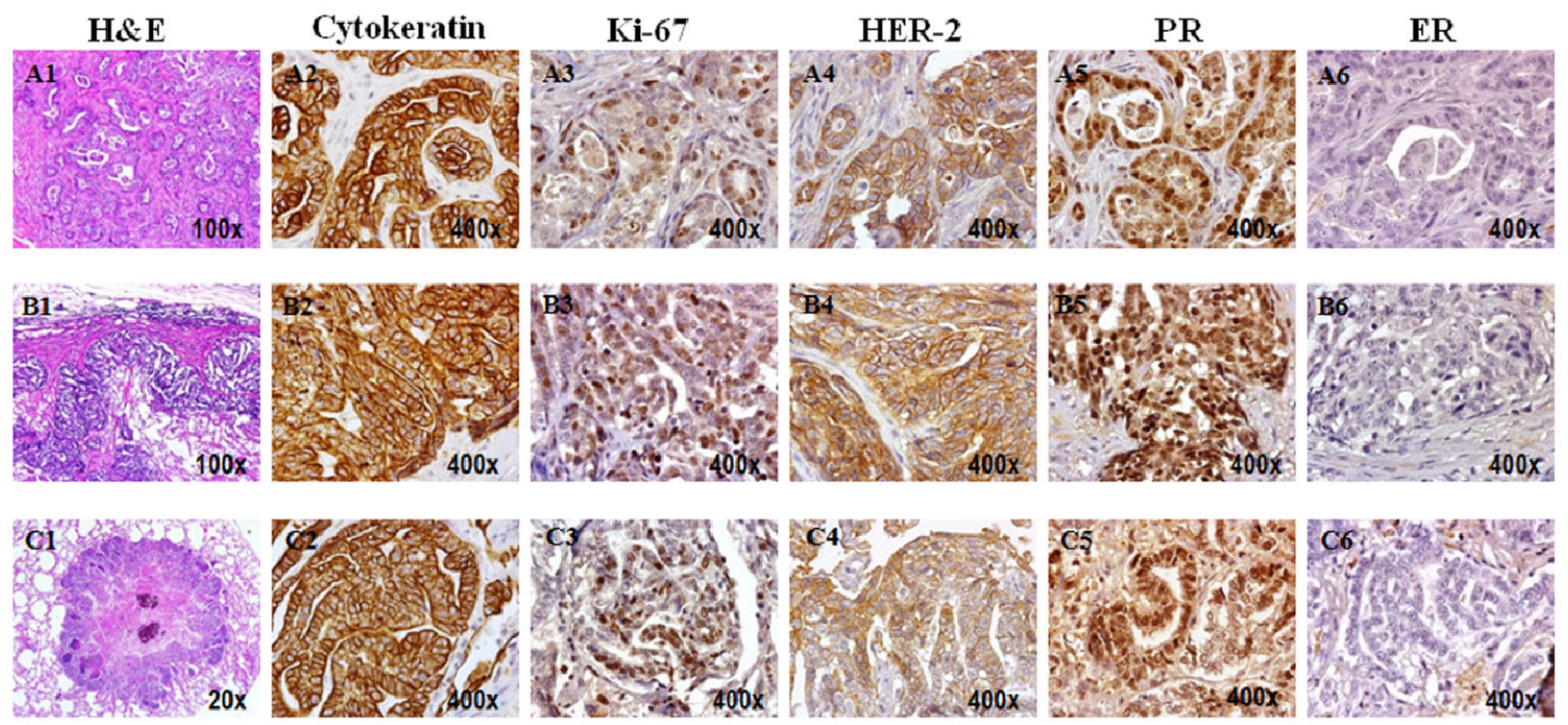

Figure 2. Immunophenotype of a feline tubulopapillary mammary carcinoma: primary tumor (A1-A6), regional metastasis (B1-B6) and distant metastasis (C1-C6). A2, B2, C2 - Intense membrane CK immunoreactivity; A3, B3, C3 - Ki-67 nuclear staining; A4, B4, C4 - HER-2 positive status; A5, B5, C5 - PR positive staining; A6, B6, C6 - ER negative staining.

The authors acknowledge the funding by Fundação para a Ciência e Tecnologia through PhD fellowship SFRH/BD/70720/2010. 